\title{
Cross-layer Design of MIMO-enabled WLANs with Network Utility Maximization
}

\author{
Yuxia Lin, Student Member, IEEE, and Vincent W.S. Wong, Senior Member, IEEE
}

\begin{abstract}
Wireless local area networks (WLANs) have become a ubiquitous high-speed data access technology. The recent IEEE 802.11e standard provides quality of service $(\mathrm{QoS})$ support, and the pending 802.11n standard further increases the transmission rate by using the multiple-input-multiple-output (MIMO) technique. Multiple antennas can be used to improve the performance gain by either increasing the transmission reliability through spatial diversity or increasing the transmission rate through spatial multiplexing. This new characteristic at the wireless physical layer requires the corresponding adaptation at the medium access control (MAC) layer to reach the best performance gain. In this paper, we propose cross-layer design schemes for WLANs under two different MAC protocols: the carrier sense multiple access with collision avoidance (CSMA/CA)-based 802.11e MAC, and the slotted Aloha MAC. For the 802.11e MAC, two different contention window size adaptation schemes, namely $U$-MAC and $D$-MAC, are proposed, which facilitate the MAC protocol to adapt its contention window size jointly with the physical layer's MIMO operating parameters. For the slotted Aloha MAC, a crosslayer optimization framework $N U M-O$ is proposed for jointly optimizing the MIMO configuration at the physical layer and the persistent probabilities for different classes of multimedia traffic at the MAC layer. A distributed algorithm NUM-D based on dual decomposition and a simplified version $N U M-S$ are also proposed. Simulation results are presented to show the effectiveness of the proposed methods.
\end{abstract}

Index Terms-WLANs, 802.11e, MIMO, slotted Aloha, network utility maximization, optimization.

\section{INTRODUCTION}

In recent years, there has been a tremendous growth in adopting the IEEE 802.11 standards for high-speed wireless local area networks (WLANs). The recent 802.11e medium access control (MAC) protocol [1] can provide differentiated quality-of-service (QoS) support via the Enhanced Distributed Channel Access (EDCA) function. In EDCA, the traffic is classified into four access categories (ACs), where each AC has different priority for channel access based on its own Arbitration Inter-Frame Space (AIFS), transmission opportunity values, and minimum and maximum contention window (CW) sizes. Among these parameters, the minimum contention window size has been shown to have a major impact on the network performance [2]. Various schemes have been proposed to tune the contention window size in order to improve the

Manuscript received November 16, 2007; revised April 17, 2008, July 9, 2008, August 25, 2008; accepted October 6, 2008. This work was supported by the Natural Sciences and Engineering Research Council (NSERC) of Canada. Part of this paper was presented at the IEEE International Conference on Communications, Beijing, China, May 2008 and IEEE Wireless Communications and Networking Conference, Las Vegas, Nevada, March/April 2008. The review of this paper was coordinated by Prof. Riku Jantti.

The authors are with the Department of Electrical and Computer Engineering, University of British Columbia, Vancouver, BC V6T 1Z4, Canada, e-mail: $\{$ yuxial, vincentw $\} @$ ece.ubc.ca.
MAC efficiency [3]. The recent 802.11 n proposal [4] aims to increase the physical link data rate up to $600 \mathrm{Mbps}$, by using the multiple-input-multiple-output (MIMO) technology at the physical layer [5]. In a wireless fading channel, two major performance gains can be realized with a wireless MIMO system: the diversity gain and spatial multiplexing gain [6].

Some recent work has studied the multiplexing and diversity gains of wireless MIMO systems under different cross-layer frameworks. A MIMO-enabled MAC protocol was proposed in [7], where the protocol mitigates interferences by utilizing the multiplexing capability of the MIMO system. Another MIMOenabled MAC protocol based on CSMA/CA in an ad-hoc network was proposed in [8], where the MAC layer schedules the transmission of different spatial streams by utilizing the spatial multiplexing capability of the MIMO system. The MIMO antenna's spatial beamforming capability is utilized in [9] by the proposed SPACE-MAC protocol.

Some other schemes allow the system to adaptively switch between the two MIMO working modes. The adaptive routing protocol in [10] directs a link to operate under full spatial diversity gain when the distance is long and the signal is weak. It operates under maximal spatial multiplexing gain when the distance is short and the signal is strong so as to increase the throughput. The transmission control protocol (TCP) over wireless MIMO channel is shown to perform better with a more reliable link provided by the diversity gain of a spacetime block coding system at low SNR regions, while a spatial multiplexing scheme outperforms in the high SNR regions [11]. All the cross-layer designs summarized above only focus on utilizing one of the two MIMO resources at a time: either full diversity or full spatial multiplexing. But it is not possible to realize both maximal diversity gain and maximal spatial multiplexing gain simultaneously. Apart from the two working modes where we can obtain a maximal multiplexing gain $r$ while the diversity gain $d$ is zero (or vice versa), there are intermediate working modes where we can achieve part of the diversity gain and multiplexing gain simultaneously. This is called the diversity-multiplexing tradeoff. It is intuitive to consider whether adaptively tuning the MIMO system is more beneficial than only choosing either the maximal multiplexing or the maximal diversity gain.

Some recent schemes investigated the possibility of achieving the diversity-multiplexing tradeoff. Lu and Kumar constructed a space-time block code to achieve any integer point on the multiplexing-diversity tradeoff curve [12]. In [13], Lee et al. studied the optimal tradeoff in a MIMO ad-hoc network by formulating the rate-reliability tradeoff problem as a network utility maximization problem. However, only one 
class of traffic is considered, and the MAC uses a reservationbased scheduling scheme, where the transmission time by each node has to be allocated in advance.

Network utility maximization (NUM) has been shown to be an effective method to tackle cross-layer optimization problems in wireless networks [14]. The utility function for each source node is usually chosen such that it reflects the satisfaction attained by the user for the services that it receives. When the NUM formulation satisfies certain conditions, such as being a separable convex optimization problem, fully distributed algorithms can be obtained, which are highly desirable in today's distributed networking environment.

In this work, we consider how to best utilize this multiplexing-diversity tradeoff in WLANs with MIMO channels. We formulate the cross-layer design as NUM problems, where the MAC layer parameters and the multiplexingdiversity tradeoff at the physical layer are jointly optimized. Two types of MAC protocols are studied in this paper, the carrier sense multiple access with collision avoidance (CSMA/CA) MAC, which is used by the 802.11e EDCA, and the slotted Aloha MAC. These are two of the most widely used random access protocols. For 802.11's CSMA/CA MAC, a centralized scheme is proposed with the access point being the centralized controller. For the slotted Aloha MAC, we construct a similar model based on the EDCA MAC model. The Aloha MAC provides us with a more accurate throughput model, which enables us to include hard QoS constraints into the NUM formulation. We further convert the Aloha MAC problem into a separable dual problem and propose a distributed algorithm to solve it.

To formulate the 802.11e-based WLAN with MIMO channels as a NUM problem, we first need to find an appropriate system model. Most of the research work in this area has focused on the saturation throughput analysis of the 802.11 WLAN, which is the maximum load that the system can carry in the saturated condition (i.e., the transmission queue of every station is assumed to be always nonempty). This is a fundamental performance figure which indicates the stable limit of the network throughput when the offered traffic load increases. Simulation studies [15] and experimental testbeds [16] have been constructed to analyze the throughput performance of EDCA. Analytical models are also proposed (e.g., [17]-[19]).

One major obstacle to utilize the above models for NUM formulation is their computational complexity, which usually involves solving a set of non-linear equations. To facilitate practical implementation, it is preferable to use a closed-form expression for the throughput in the utility optimization problem. As a result, in this paper, we propose two 802.11e EDCA throughput models based on several simplifying assumptions. These assumptions provide us an EDCA model where the saturation throughput can be derived with a closed-form solution. This greatly facilitates the NUM formulation, and improves the convergence speed of the optimization problem. Although the simplifying assumptions may reduce the accuracy of the model, simulation results show the effectiveness of the proposed schemes, and verify that the simplifying assumptions provide a good trade-off to obtain the solutions.

The contributions of our work are as follows:
1) For 802.11e WLANs with MIMO channels, we propose two cross-layer schemes, namely $U-M A C$ and $D-M A C$, which jointly select the MIMO coding scheme at the physical layer and the minimum contention window size at the MAC layer to achieve the maximal network utility.

2) For the slotted Aloha based WLAN, we propose a crosslayer design framework $N U M-O$ which jointly selects the MIMO coding scheme at the physical layer and the transmission persistent probability at the MAC layer to achieve the maximal network utility. By using the dual decomposition method, we then propose a distributed algorithm $N U M-D$. We further propose another algorithm called $N U M-S$, which is also practical for implementation.

3) Simulation results are presented to study the effectiveness of the proposed schemes. The $U-M A C$ and $D-M A C$ schemes are compared with the original 802.11e EDCA protocol under MIMO channels. Both system throughput and delay performance are shown to improve significantly. For the slotted-Aloha MAC, network throughput and reliability performance of the distributed $N U M-D$ scheme is compared to the NUM-S scheme.

The comparison between our proposed algorithms is shown in Table I. The rest of the paper is organized as follows. System models and the cross-layer optimization schemes $U-M A C$ and $D-M A C$ for the 802.11e WLAN are presented in Section II. Section III proposes the system model $N U M-O$ for the slotted Aloha based WLAN, and its distributed solution NUM-D. Simulation results are presented in Section IV. Conclusions and future work are given in Section V.

\section{System Model For 802.11E EDCA MAC}

Consider a WLAN with an access point (AP). Let $\mathcal{N}$ denote the set of wireless stations. According to the EDCA specifications in the 802.11 e standard, there can be up to four different classes of traffic with different QoS requirements. For simplicity, we study the case of two access categories $\left(A C_{1}\right.$ and $A C_{2}$ ) with $A C_{2}$ being the higher priority traffic in the network. We use $\mathcal{N}_{1}$ and $\mathcal{N}_{2}$ to denote the set of wireless stations in $A C_{1}$ and $A C_{2}$, respectively. Each wireless station transmits one class of traffic, so the set of all stations $\mathcal{N}$ is $\mathcal{N}_{1} \cup \mathcal{N}_{2}$. We use $N_{1}, N_{2}$, and $N$ to denote the number of stations in the set. Thus, $N_{1}=\left|\mathcal{N}_{1}\right|, N_{2}=\left|\mathcal{N}_{2}\right|$, and $N=|\mathcal{N}| . N_{1}, N_{2}$ and $N$ are assumed to be known constants in the system model. Estimation of the number of competing nodes in the network has been studied in [20], [21], and is beyond the scope of this paper. Extension to four access categories is fairly straightforward based on the optimization framework proposed below.

We study the performance of wireless stations competing for channel access and sending data to the AP. Each wireless station has $M_{T}$ transmit antennas, and the AP has $M_{R}$ receive antennas. There is a fundamental tradeoff between a MIMO system's multiplexing and diversity gains. For each spatial multiplexing gain $r$, the best diversity gain $d^{*}(r)$ is the supremum of the diversity gain achieved over all schemes. In a Rayleigh-fading channel with long enough block lengths, 
TABLE I

Comparison between Proposed Schemes

\begin{tabular}{|c|c|c|c|c|c|c|}
\hline Scheme & Operation & MAC & Input Parameters & Variables & $\begin{array}{c}\text { Output } \\
\text { Solutions }\end{array}$ & Comments \\
\hline$U-M A C$ & Centralized & CSMA/CA & $\begin{array}{c}M_{T}, M_{R}, \alpha, \beta, \mathcal{N}, L \\
k_{c}, k_{p}, \gamma, T_{h d r}, T_{A C K} \\
T_{\text {slot }}, T_{S I F S}, T_{A I F S}\end{array}$ & $\mathbf{x}, \mathbf{y}, \mathbf{r}, \bar{W}_{a}$ & $\begin{array}{l}\text { PHY: } \mathbf{r} \\
\text { MAC: } \bar{W}_{a}\end{array}$ & $\begin{array}{l}\text { Uniform } \mathrm{CW} \\
\text { for each } A C \text {. }\end{array}$ \\
\hline$D-M A C$ & Centralized & CSMA/CA & $\begin{array}{c}M_{T}, M_{R}, \alpha, \beta, \mathcal{N}, L \\
k_{c}, k_{p}, \gamma, T_{h d r}, T_{A C K} \\
T_{\text {slot }}, T_{S I F S}, T_{A I F S}\end{array}$ & $\mathbf{x}, \mathbf{y}, \mathbf{r}, W_{s}$ & $\begin{array}{c}\text { PHY: } \mathbf{r} \\
\text { MAC: } W_{s}\end{array}$ & $\begin{array}{l}\text { Differentiated CW } \\
\text { for each station. }\end{array}$ \\
\hline$N U M-O$ & Centralized & Slotted Aloha & $\begin{array}{c}M_{T}, M_{R}, \alpha, \beta, \mathcal{N} \\
k_{c}, k_{p}, \gamma, R\end{array}$ & $\mathbf{x}, \mathbf{y}, \mathbf{r}, \mathbf{p}$ & $\begin{array}{l}\text { PHY: } \mathbf{r} \\
\text { MAC: } \mathbf{p}\end{array}$ & $\begin{array}{l}\text { Centralized NUM } \\
\text { formulation. }\end{array}$ \\
\hline$N U M-D$ & Distributed & Slotted Aloha & $\begin{array}{c}M_{T}, M_{R}, \alpha, \beta, \mathcal{N} \\
k_{c}, k_{p}, \gamma, R\end{array}$ & $\begin{array}{c}\mathbf{x}^{\prime}, \mathbf{y}, \mathbf{r} \\
\mathrm{p}^{\prime}, \mathrm{p}^{\prime \prime}, \lambda, \mu\end{array}$ & $\begin{array}{l}\text { PHY: } \mathbf{r} \\
\text { MAC: } \mathbf{p}\end{array}$ & $\begin{array}{l}\text { Distributed solution } \\
\text { for } N U M-O .\end{array}$ \\
\hline$N U M-S$ & Distributed & Slotted Aloha & $\begin{array}{c}M_{T}, M_{R}, \alpha, \beta, \mathcal{N} \\
k_{c}, k_{p}, \gamma, R\end{array}$ & $\mathbf{x}, \mathbf{y}, \mathbf{r}, \mathbf{p}$ & $\begin{array}{l}\text { PHY: } \mathbf{r} \\
\text { MAC: } \mathbf{p}\end{array}$ & $\begin{array}{l}\text { Simplified scheme as } \\
\text { performance baseline. }\end{array}$ \\
\hline
\end{tabular}

the optimal multiplexing-diversity tradeoff $d^{*}(r)$ is given by the piecewise-linear function connecting the points $\left(r, d^{*}(r)\right)$, for $r=0,1, \ldots, \min \left(M_{T}, M_{R}\right)$ [22]:

$$
d^{*}(r)=\left(M_{T}-r\right)\left(M_{R}-r\right),
$$

where $M_{T}$ and $M_{R}$ are the number of transmit and receive antennas, respectively. In this paper, we assume that a family of carefully designed codes can achieve the above optimal tradeoff performance. However, this curve is nondifferentiable, which may cause difficulties to solve the NUM problem. We use the differentiable approximation as in [13]:

$$
d(r)=\left(M_{T}-r\right)\left(M_{R}-r\right), \quad 0 \leq r \leq \min \left(M_{T}, M_{R}\right) .
$$

This differentiable approximation is a lower bound of (1), which gives a subset of the feasible diversity-multiplexing tradeoff region. Although this can potentially lead to a suboptimal solution of the problem due to the reduced tradeoff region, as analyzed in [13], this approximation is close to the exact tradeoff relationship and the reduction of the feasible tradeoff region is small. Thus, the impact on the results should not be significant.

If a wireless link from the wireless station $s \in \mathcal{N}$ to the AP has the average signal-to-noise ratio $\gamma_{s}$, multiplexing gain $r_{s}$ and diversity gain $d_{s}$, then the link data rate $c_{s}\left(\gamma_{s}\right)$ (bps) and error probability $p_{s}^{e r r}\left(\gamma_{s}\right)$ can be approximated as [23]:

$$
c_{s}\left(\gamma_{s}\right)=k_{c} r_{s} \log \gamma_{s},
$$

and

$$
p_{s}^{e r r}\left(\gamma_{s}\right)=k_{p} \gamma_{s}^{-d\left(r_{s}\right)},
$$

where $k_{c}$ and $k_{p}$ are positive constants for different coding schemes. The log function uses base 2 throughout this paper. The multiplexing gain $r_{s}$ and diversity gain $d_{s}$ conform to the optimal tradeoff in (2). Then, the link transmission reliability $y_{s}$ is:

$$
y_{s}=1-k_{p} \gamma_{s}^{-d\left(r_{s}\right)}, \quad s \in \mathcal{N} .
$$

With $c_{s}$ being the data rate of wireless station $s$, the achievable throughput of each station is usually significantly lower than $c_{s}$ due to the random access and the protocol overhead [2]. To formulate the NUM problem, we need an analytical model for the throughput of each station with respect to $c_{s}$ and other network parameters, which is a difficult task due to the CSMA/CA based access scheme. However, the saturation throughput is more tractable and has been successfully derived from various mathematical models.

For a single cell 802.11 WLAN, its saturation throughput is a good indicator of network performance because it is the sustainable throughput under heavy traffic load, and is also close to the maximum network capacity. In this work, we base our analysis on the saturation network performance. There are two causes of packet loss in an 802.11 WLAN. One is the packet collision where two nodes transmit simultaneously. The other one is channel error, where a packet is received without packet collisions, but is corrupted due to low SNR.

In this paper, we denote the saturation throughput under an ideal channel (i.e., no channel errors) as $x_{s}$. Then, $x_{s} y_{s}$ can approximately represent the effective throughput after further discarding the corrupted packets due to channel errors. This approximation separates the effects of packet collision and channel error on the throughput calculation, and greatly facilitates the problem formulation.

The throughput $x_{s}$ is primarily a function of the physical layer parameters, such as the network basic and data rates, and the MAC layer parameters such as contention window sizes, the DIFS/SIFS/AIFS values, and the frame header overhead. Of all the elements listed above, we focus on studying the effects of the data rate $c_{s}$ and the minimum contention window size $W_{s}$ for station $s$ in this paper. The data rate with a MIMO channel is given by (3), and it is a function of the SNR $\gamma_{s}$ and the MIMO operation mode $r_{s}$.

We consider two cases of the assignment of $W_{s}$. In the first case, there is a uniform $\mathrm{CW}$ value for each $\mathrm{AC}$, where all wireless stations belonging to the same AC use the same minimum contention window value. This is the normal behavior in 802.11e. We call this MAC as the Uniform-CW MAC or $U$-MAC. In the second case, we allow each wireless station to freely choose its own minimum contention window size without conforming to the same $\mathrm{CW}$ in its $\mathrm{AC}$. We call this MAC as the Differentiated-CW MAC or D-MAC.

Time is partitioned into virtual time slots where each slot can be either a backoff time slot, a collision-free transmission slot, or a collided transmission slot. The probability for station $s$ to transmit in a virtual time slot can be approximated as 
follows [24]:

$$
\tau_{s}=\frac{2}{W_{s}+1}, \quad s \in \mathcal{N},
$$

where $W_{s}$ is the minimum contention window size for station $s$. The probability that one transmitted packet by station $s$ will collide is the probability that any of the other stations also transmit:

$$
q_{s}=1-\prod_{k \in \mathcal{N}, k \neq s}\left(1-\tau_{k}\right), \quad s \in \mathcal{N} .
$$

In the following subsections, we describe how to determine the throughput $x_{s}$ for the two MAC schemes separately below.

\section{A. Saturation Throughput Analysis of the U-MAC Scheme}

For the $U-M A C, \tau_{s}$ and $q_{s}$ in (6) and (7) are reduced to the same values for each $\mathrm{AC}$, as we require that each $\mathrm{AC}$ has a uniform minimum contention window size, which is $W_{s}=\bar{W}_{a}$, for wireless station $s$ transmitting $A C_{a}$ traffic. Thus from (6), we can use $\tau_{a}$ to represent any $\tau_{s}, s \in \mathcal{N}_{a}$, for $a=1,2$. To calculate $x_{s}$, we extend the mean value based saturation throughput analysis in [25]. The main difference here is that the wireless stations may use different data rates other than the uniform data rate assumed in [25]. The average length of one virtual time slot $T_{a}$ for $A C_{a}$ (where $a=1,2$ ) can be approximated as follows:

$$
\begin{aligned}
T_{a}= & \sum_{s \in \mathcal{N}_{a}} \frac{\tau_{s}}{\sum_{k \in \mathcal{N}_{a}} \tau_{k}} \frac{L}{c_{s}}+T_{h d r}+T_{S I F S}+T_{A C K} \\
& +T_{A I F S}+\frac{\bar{W}_{a}}{N_{a}+1} T_{\text {slot }}, \quad a=1,2,
\end{aligned}
$$

where $T_{h d r}, T_{A C K}, T_{\text {slot }}, T_{S I F S}$ and $T_{A I F S}$ denote the length of the frame header, the ACK frame, the backoff time slot length, the SIFS and AIFS length, respectively. $\bar{W}_{a}$ (for $a=1,2$ ) represents the minimum contention window size of $A C_{a}$. This paper focuses on studying the effects of contention window size adaptation, and thus the same AIFS is used for each AC. An identical payload length $L$ is assumed for the network. $L / c_{s}$ is the time to transmit the frame payload of station $s$. The average payload transmission time is the weighted sum of all $A C_{a}$ stations, using each station's transmission probability as the weight.

From [25], we can calculate the rate of success for $A C_{a}$, which is the average number of collision-free transmissions by $A C_{a}$ per second, as follows:

$$
v_{a}=\frac{N_{a} \tau_{a}\left(1-q_{a}\right)}{\left(1-q_{1} / 2\right) N_{1} \tau_{1} T_{1}+\left(1-q_{2} / 2\right) N_{2} \tau_{2} T_{2}}, a=1,2 .
$$

The aggregated saturation throughput of all stations belonging to $A C_{a}$ is equal to $v_{a} L$. The saturation throughput for station $s$ in each AC can be calculated as follows:

$$
\hat{x}_{s}=v_{a} L \frac{c_{s}\left(\gamma_{s}\right)}{\sum_{i \in \mathcal{N}_{a}} c_{i}\left(\gamma_{i}\right)}, \quad \forall s \in \mathcal{N}_{a}, \quad a=1,2 .
$$

The above equation shows that the individual saturation throughput of each station $s$ in $A C_{a}$ is the weighted portion of $v_{a} L$ in proportion to its data rate $c_{s}$.

\section{B. Saturation Throughput Analysis of the D-MAC Scheme}

For the $D-M A C$, we allow each station $s$ to choose its own $W_{s}$ value individually based on its link state and class of traffic. In this case, we have an 802.11e WLAN with multiple data rates, and multiple $\mathrm{CW}$ values. To model this system accurately is a challenging task. Even for the basic 802.11e MAC with a single data rate, complicated multi-dimensional Markov chain and queueing models have to be used, which result in complex systems of non-linear equations to solve for the throughput $x_{s}$ [18]. Although these models give accurate results, they usually require some stringent assumptions, and none can handle a versatile system where each wireless station has a distinct data rate and a distinct $\mathrm{CW}$.

However, we may not need a highly accurate throughput model for the NUM framework to work properly. Other factors, such as the convergence speed of the optimization problem and the computation efficiency, are important for a NUM problem. It is preferable to obtain a closed form expression of $x_{s}$ instead of a system of nonlinear equations on $x_{s}$. With this goal, we propose that the throughput of station $s$ be modeled as:

$$
\hat{x}_{s}=\Gamma \tau_{s}\left(1-q_{s}\right) \frac{L}{T_{a}}, \quad s \in \mathcal{N}_{a}, \quad a=1,2,
$$

where $\Gamma$ is a positive scaling factor. Here, we assume that the two ACs have the same scaling factor. In Section II-D, we can see that the exact value of $\Gamma$ need not be known for solving our NUM problem. By extending the model from [25] with approximating the uniform contention window size with $\sum_{s \in \mathcal{N}_{a}} W_{s} / N_{a}$, the average virtual slot length $T_{a}$ (for $a=$ $1,2)$ is defined as follows:

$$
\begin{aligned}
T_{a}= & \sum_{s \in \mathcal{N}_{a}} \frac{\tau_{s}}{\sum_{k \in \mathcal{N}_{a}} \tau_{k}} \frac{L}{c_{s}}+T_{h d r}+T_{S I F S}+T_{A C K} \\
& +T_{A I F S}+\frac{\sum_{s \in \mathcal{N}_{a}} W_{s}}{N_{a}\left(N_{a}+1\right)} T_{\text {slot }}, \quad a=1,2,
\end{aligned}
$$

where we use the average contention window size $\sum_{s \in \mathcal{N}_{a}} W_{s} / N_{a}$ as an approximation of the identical contention window size modeled in [25].

Equation (11) states that the throughput of wireless station $s$ is in proportion to its transmission probability $\tau_{s}$, collisionfree probability $\left(1-q_{s}\right)$, and the average transmission rate of one payload by each $A C_{a}\left(L / T_{a}\right)$. This is a reasonable throughput approximation as the transmission and collision probabilities of a station are the major factors influencing its throughput. The linear proportional relationship is a bold assumption. From our later simulation results, we can verify that our NUM formulation based on this simple assumption can efficiently achieve the desired traffic differentiation and utility optimization effects.

\section{Network Utility Formulation}

We use the following $\alpha$-fair utility function [26]:

$$
U(x)= \begin{cases}(1-\alpha)^{-1} x^{(1-\alpha)}, & \text { if } \alpha \in(0,1) \cup(1, \infty), \\ \log x, & \text { if } \alpha=1,\end{cases}
$$


where $\alpha$ is the fairness parameter. If $x$ is the throughput of the source node, then $\alpha \rightarrow 0$ leads to throughput maximization. Proportional and harmonic mean fairness are achieved when $\alpha=1$ and $\alpha=2$, respectively. When $\alpha \rightarrow \infty$, max-min fairness can be achieved. In our NUM framework, we choose the utility function for each wireless station $s$ with $\alpha>1$ :

$$
U_{a}\left(x_{s}, y_{s}\right)=(1-\alpha)^{-1}\left(x_{s} y_{s}\right)^{1-\alpha}, \quad \alpha>1, \quad a=1,2
$$

where $x_{s} y_{s}$ is the effective throughput of station $s$ after accounting for both packet collisions and channel errors. $U_{a}$ represents the utility function of $A C_{a}$ for $a=1$ and 2 .

The network utility function is defined as:

$$
\begin{aligned}
U(\mathbf{x}, \mathbf{y})= & \sum_{i \in \mathcal{N}_{1}} \beta U_{1}\left(x_{i}, y_{i}\right) \\
& +\sum_{j \in \mathcal{N}_{2}}(1-\beta) U_{2}\left(x_{j}, y_{j}\right), \quad \alpha>1,
\end{aligned}
$$

where $\beta$ is an adjustable parameter between 0 and 0.5 to tune the weight of $A C_{1}$ and $A C_{2}$ on the network utility. It can be proven that $U(\mathbf{x}, \mathbf{y})$ is a concave function of $\mathbf{x}, \mathbf{y}$.

\section{NUM Framework for Cross-layer Design}

With the above system model and definition of the network utility, we propose our NUM formulation for crosslayer design of a MIMO-enabled 802.11e WLAN, where each station's MIMO multiplexing gain at the physical layer and the contention window sizes at the MAC layer are jointly optimized.

1) U-MAC: Wireless stations use the minimum contention window size of $W_{a}$ in $A C_{a}$. The NUM problem can be formulated as follows:

$$
\begin{array}{ll}
\max _{\mathbf{x}, \mathbf{y}} & \sum_{i \in \mathcal{N}_{1}} \beta U_{1}\left(x_{i}, y_{i}\right)+\sum_{j \in \mathcal{N}_{2}}(1-\beta) U_{2}\left(x_{j}, y_{j}\right) \\
\text { st } \quad & 0 \leq x_{s} \leq v_{a} L \frac{c_{s}\left(\gamma_{s}\right)}{\sum_{n \in \mathcal{N}_{a}} c_{n}\left(\gamma_{n}\right)}, \forall s \in \mathcal{N}_{a}, a=1,2 \\
& \check{y}_{a} \leq y_{s} \leq 1-k_{p} \gamma_{s}^{-\left(M_{T}-r_{s}\right)\left(M_{R}-r_{s}\right)}, \\
& \forall s \in \mathcal{N}_{a}, a=1,2 \\
& 0 \leq r_{s} \leq \min \left(M_{T}, M_{R}\right), \quad \forall s \in \mathcal{N} \\
& \check{W}_{a} \leq \bar{W}_{a} \leq \bar{W}_{a}, \quad a=1,2,
\end{array}
$$

where $v_{a}$ is given by (9), and is a function of $\bar{W}_{a}, r_{s}$ and other system parameters. The parameters $\check{y}_{a}, \bar{W}_{a}$ and $\bar{W}_{a}$ are constant bounds on the variables, and should be selected within reasonable ranges. The above problem is a NUM problem with variables $\mathbf{x}, \mathbf{y}, \mathbf{r}$ and $\bar{W}_{a}$. The saturation throughput from (10) is used as the upperbound for $x_{s}$.

Although the objective function in (16) is concave, the constraint sets make this problem non-convex and challenging to solve. However, our tests with Matlab's nonlinear optimization toolbox, using the sequential quadratic programming (SQP) method [27], show that this problem formulation, under reasonable ranges of network parameters and initial variable values, has no difficulty in converging to a unique solution. Results from Section IV show that the number of iterations needed is in the range of 20 to 200 . Note that due to nonconvexity of the problem, the solution can be sub-optimal in the sense that a local instead of the global optimal solution is determined. When the link SNR values drop below certain levels, the problem may no longer have a feasible solution. Analytically deriving the feasible region of the NUM problem is difficult. However, from an engineering point of view, whenever a feasible solution is unavailable, it means that one or more of the stations with the lowest SNRs will not satisfy the minimum QoS requirements. There are two possible actions to take: reducing the QoS demand or disassociating those stations from the network. To study these scenarios in detail is beyond the scope of the current paper and will be subject of our future study.

2) $D$-MAC: With each station $s$ choosing its minimum contention window size $W_{s}$ individually, the NUM problem can be formulated as follows:

$$
\begin{aligned}
& \max _{\mathbf{x}, \mathbf{y}} \sum_{i \in \mathcal{N}_{1}} \beta U_{1}\left(x_{i}, y_{i}\right)+\sum_{j \in \mathcal{N}_{2}}(1-\beta) U_{2}\left(x_{j}, y_{j}\right) \\
& \text { st } \quad 0 \leq x_{s} \leq \Gamma \tau_{s}\left(1-q_{s}\right) \frac{L}{T_{a}}, \quad \forall s \in \mathcal{N}_{a}, \quad a=1,2 \\
& \check{y}_{a} \leq y_{s} \leq 1-k_{p} \gamma_{s}^{-\left(M_{T}-r_{s}\right)\left(M_{R}-r_{s}\right)} \\
& \forall s \in \mathcal{N}_{a}, \quad a=1,2 \\
& 0 \leq r_{s} \leq \min \left(M_{T}, M_{R}\right), \quad \forall s \in \mathcal{N} \\
& \check{W}_{s} \leq W_{s} \leq \hat{W}_{s}, \quad \forall s \in \mathcal{N},
\end{aligned}
$$

where $\hat{W}_{s}$ and $\check{W}_{s}$ are the lower and upper bounds of the contention window for station $s$, respectively. The variables $\tau_{s}, q_{s}$, and $T_{a}$ are given in (6), (7) and (12). Note that for the constant parameter $\Gamma$, it ends up being a scaling factor $\Gamma^{1-\alpha}$ in the objective function as defined by (15), and has no effect on the optimal solutions, which are the system's minimum contention window size $W_{s}$ and the MIMO multiplexing gain $r_{s}$. The above problem is a NUM problem with variables $\mathbf{x}$, $\mathbf{y}, \mathbf{r}$, and $W_{s}$. It can also be solved using standard algorithms for constrained nonlinear optimization problems as discussed in Section II-D.1.

With the above formulation, the AP in an 802.11e WLAN can collect each station's link SNR, select a $\beta$ with appropriate network revenue models, and solve either (16) or (17) to obtain the MIMO multiplexing gain $r_{s}$ and minimum contention window size $W_{s}$ for each station. The resulting $r_{s}$ and $W_{s}$ values can be sent out in the EDCA parameter set elements of the 802.11 e beacon frame for each station to adjust its physical and MAC layer operation parameters.

\section{System Model for Slotted Aloha MAC}

In this section, we consider the slotted Aloha MAC. The NUM formulation can be transformed to a separable convex optimization problem, and a distributed algorithm can be derived accordingly. For the slotted Aloha, we can obtain the throughput formulation without assuming the saturated conditions. When source node $s$ transmits with persistent probability $p_{s}$ in slotted Aloha, its throughput is:

$$
x_{s}=c_{s}\left(\gamma_{s}\right) p_{s} \prod_{k \in \mathcal{N}, k \neq s}\left(1-p_{k}\right), \quad s \in \mathcal{N} .
$$

The transmission reliability $y_{s}$ is the same as in (5). Instead of only differentiating the priority of $A C_{1}$ and $A C_{2}$ traffic, we 
further assume that the higher priority traffic $\left(A C_{2}\right)$ is realtime traffic with constant-bit-rate (CBR) flows, which has a hard throughput requirement $R$ and reliability demand $Q$ :

$$
\begin{array}{ll}
R \leq x_{j} \leq \hat{x}=k_{c} \hat{r} \log \hat{\gamma}, & j \in \mathcal{N}_{2}, \\
Q \leq y_{j} \leq 1, & j \in \mathcal{N}_{2},
\end{array}
$$

where $\hat{x}$ is the constant upper bound on throughput, with $\hat{r}$ and $\hat{\gamma}$ being the network's maximum multiplexing gain and maximum SNR value, respectively. The maximum SNR value can either be deduced from the network channel condition under study or be assigned a high enough value to put a reasonable upper bound in the problem formulation.

The $A C_{1}$ traffic is best-effort traffic, and also has the bounding constraints:

$$
\begin{aligned}
& \check{x}_{1} \leq x_{i} \leq \hat{x}, \\
& \check{y}_{1} \leq y_{i} \leq 1,
\end{aligned}
$$

where $\check{x}_{1}$ and $\check{y}_{1}$ are the constant lower bound on throughput and reliability for best-effort traffic, respectively.

\section{A. Network Utility Formulation}

To differentiate the different QoS requirement of $A C_{1}$ and $A C_{2}$, we use the utility function of (13) with $\alpha>1$, and extend it to include a normalizing denominator. Thus, the utility function for the wireless stations with $A C_{1}$ traffic is as follows:

$$
U_{1}\left(x_{i}, y_{i}\right)=\frac{\left(x_{i} y_{i}\right)^{1-\alpha}-\left(\check{x}_{1} \check{y}_{1}\right)^{1-\alpha}}{(\hat{x})^{1-\alpha}-\left(\check{x}_{1} \check{y}_{1}\right)^{1-\alpha}}, \quad \alpha>1
$$

where the product $x_{i} y_{i}$ can be interpreted as the effective throughput for the best-effort station $i \in \mathcal{N}_{1}$.

For the CBR traffic in $A C_{2}$, the utility function is chosen to be a function of the reliability only, because we expect that the throughput $x$ will satisfy the CBR traffic's throughput demand $R$ as in (19); however, further increasing a CBR traffic's throughput provisioning usually does not provide additional performance gains:

$$
U_{2}\left(y_{i}\right)=\frac{y_{i}^{1-\alpha}-Q^{1-\alpha}}{1-Q^{1-\alpha}}, \quad \alpha>1 .
$$

The utility functions in (21) and (22) are normalized such that $U_{1}\left(\check{x}_{1}, \check{y}_{1}\right)=U_{2}(Q)=0$ and $U_{1}(\hat{x}, 1)=U_{2}(1)=1$. This avoids the apparent problem of combining two utility functions, which would otherwise have a few magnitude of difference in value. The network utility is the sum of the utilities of all the source nodes:

$$
\begin{aligned}
U(\mathbf{x}, \mathbf{y})= & \sum_{i \in \mathcal{N}_{1}} \beta U_{1}\left(x_{i}, y_{i}\right) \\
& +\sum_{j \in \mathcal{N}_{2}}(1-\beta) U_{2}\left(y_{j}\right), \quad \alpha>1
\end{aligned}
$$

where $\mathbf{x}, \mathbf{y}$ are vectors for each source's throughput and reliability, $\beta$ is an adjustable parameter between 0 and 1 to tune the weight of $A C_{1}$ and $A C_{2}$ 's utility values. It can be shown that $U(\mathbf{x}, \mathbf{y})$ is a concave function.

\section{B. Utility-Optimal PHY/MAC Cross-layer Design}

1) Centralized Scheme: NUM-O: We now present the centralized NUM formulation for the slotted Aloha MAC, which is called $N U M-O$. Assuming that each station $s$ can adaptively adjust its persistent probability $p_{s}$ at the MAC layer with crosslayer information from the MIMO-enabled physical layer, we propose the following utility maximization formulation:

$$
\begin{array}{lll}
\max _{\mathbf{x}, \mathbf{y}} & \sum_{i \in \mathcal{N}_{1}} \beta U_{1}\left(x_{i}, y_{i}\right)+\sum_{j \in \mathcal{N}_{2}}(1-\beta) U_{2}\left(y_{j}\right) & \\
\text { st } & x_{s} \leq k_{c} r_{s} \log \left(\gamma_{s}\right) p_{s} \prod_{k \in \mathcal{N} \backslash\{s\}}\left(1-p_{k}\right), \quad \forall s \in \mathcal{N} \\
& & \\
& y_{s} \leq 1-k_{p} \gamma_{s}^{-\left(M_{T}-r_{s}\right)\left(M_{R}-r_{s}\right)}, & \forall s \in \mathcal{N} \\
& \check{x}_{1} \leq x_{i} \leq \hat{x}, & \forall i \in \mathcal{N}_{1} \\
& R \leq x_{j} \leq \hat{x}, & \forall i \in \mathcal{N}_{2} \\
& \check{y}_{1} \leq y_{i} \leq 1, & \forall j \in \mathcal{N}_{1} \\
& Q \leq y_{j} \leq 1, & \forall s \in \mathcal{N} \\
0 \leq r_{s} \leq \min \left(M_{T}, M_{R}\right), & \forall s \in \mathcal{N} . \\
0 \leq p_{s} \leq 1, &
\end{array}
$$

Problem (24) is a non-linear optimization problem with respect to the variables $\{\mathbf{x}, \mathbf{y}, \mathbf{r}, \mathbf{p}\}$. We can use a change of variables to make the problem separable into sub-problems, and prove that the resulting problem is convex. We perform a $\log$ transformation of variables: $x_{s}^{\prime}=\log x_{s}, p_{s}^{\prime}=\log p_{s}$ and $p_{s}^{\prime \prime}=\log \left(1-p_{s}\right)$ for $s \in \mathcal{N}$ by taking $\log$ on both sides of the first constraint. The NUM problem becomes:

$$
\begin{array}{lll}
\underset{\mathbf{x}^{\prime}, \mathbf{y}}{\max } & \sum_{i \in \mathcal{N}_{1}} \beta U_{1}^{\prime}\left(x_{i}^{\prime}, y_{i}\right)+\sum_{j \in \mathcal{N}_{2}}(1-\beta) U_{2}\left(y_{j}\right) & \\
\text { st } & x_{s}^{\prime}-\log k_{c}-\log r_{s}-\log \left(\log \gamma_{s}\right) & \\
& -p_{s}^{\prime}-\sum_{k \in \mathcal{N} \backslash\{s\}} p_{k}^{\prime \prime} \leq 0, & \forall s \in \mathcal{N} \\
& & \\
& y_{s}-1+k_{p} \gamma_{s}^{-\left(M_{T}-r_{s}\right)\left(M_{R}-r_{s}\right)} \leq 0, & \forall s \in \mathcal{N} \\
& e^{p_{s}^{\prime}+e^{p_{s}^{\prime \prime}} \leq 1,} & \forall s \in \mathcal{N} \\
& \log \check{x}_{1} \leq x_{i}^{\prime} \leq \log \hat{x}, & \forall i \in \mathcal{N}_{1} \\
& \log R \leq x_{j}^{\prime} \leq \log \hat{x}, & \forall j \in \mathcal{N}_{2} \\
& \check{y}_{1} \leq y_{i} \leq 1, & \forall i \in \mathcal{N}_{1} \\
Q \leq y_{j} \leq 1, & \forall j \in \mathcal{N}_{2} \\
& 0 \leq r_{s} \leq \Delta_{s}, \quad p_{s}^{\prime}, p_{s}^{\prime \prime} \leq 0, \quad \forall s \in \mathcal{N} & (25)
\end{array}
$$

where

$$
\Delta_{s}=\min \left\{\min \left\{M_{T}, M_{R}\right\}, \frac{M_{T}+M_{R}}{2}-\frac{1}{2} \sqrt{\frac{2}{\ln \gamma_{s}}}\right\},
$$

and

$$
U_{1}^{\prime}\left(x^{\prime}, y\right)=\frac{\left(e^{x^{\prime}} y\right)^{1-\alpha}-\left(\check{x}_{1} \check{y}_{1}\right)^{1-\alpha}}{(\hat{x})^{1-\alpha}-\left(\check{x}_{1} \check{y}_{1}\right)^{1-\alpha}},
$$

The above problem is a convex optimization problem. The detailed proof of convexity is included in the Appendix. Appendices A and B show the proofs for the convexity of the objective function and the constraints, respectively. In order to obtain a convex problem, we introduced an extra constraint on $r_{s}$ as shown in (26). This constraint can reduce 
$r_{s}$ 's range of values in low SNR conditions. However, this reduction in the range of $r_{s}$ is not significant. For example, when $M_{T}=2, M_{R}=3$, and $\gamma_{s}=7 \mathrm{~dB}$, the range of $r_{s}$ is reduced from $[0,2]$ in $(24)$ to $[0,1.993]$ in $(26)$. When $\gamma_{s}$ is higher than $8 \mathrm{~dB}$, there is no longer any difference between the formulations in (24) and (25). As a result, we can expect that the transformed convex formulation (25) would obtain the same optimal solution as before the variable transformation in most cases. The possibility of obtaining sub-optimal solutions in some low SNR cases is a trade-off in order to formulate the problem as a convex optimization problem.

In a WLAN where all the wireless stations communicate with the AP directly, the AP can measure the $\operatorname{SNR}\left(\gamma_{s}\right)$ of each link and use this information to solve the persistent probability $p_{s}$ at the MAC layer, and the multiplexing gain $r_{s}$ at the MIMO physical layer. The resulting $r_{s}$ and $p_{s}$ values can be sent to each wireless station via piggyback on the control frames. Each station will then tune its operating parameters accordingly. Furthermore, the AP can adjust the parameter $\beta$ in the network utility function to adaptively tune the tradeoff between enhancing the throughput of best-effort traffic and the reliability of real-time flows. The NUM formulation also faces the problem of non-feasibility when some link SNRs are too low. We may either reduce the QoS demand or disassociating weak stations from the network to reach a feasible solution.

2) Distributed Scheme NUM-D: The NUM-O problem proposed in (24) is a centralized scheme, where the AP acts as the central controller and distributes the control information to each wireless station. This may suffer from a single-point failure. An alternative would be a fully distributed algorithm, where each wireless station can decide its own operation parameters based on the locally available information and limited message exchanges. Since problem (25) is convex, the dual decomposition approach can be used to obtain the distributed solution. By relaxing the first two constraints, the Lagrangian function for problem (25) is:

$$
\begin{aligned}
& L\left(\mathbf{x}^{\prime}, \mathbf{y}, \mathbf{r}, \mathbf{p}^{\prime}, \mathbf{p}^{\prime \prime}, \boldsymbol{\lambda}, \boldsymbol{\mu}\right) \\
& =\sum_{i \in \mathcal{N}_{1}} \beta U_{1}^{\prime}\left(x_{i}^{\prime}, y_{i}\right)+\sum_{j \in \mathcal{N}_{2}}(1-\beta) U_{2}\left(y_{j}\right) \\
& \quad+\sum_{s \in \mathcal{N}} \lambda_{s}\left(\log k_{c}+\log r_{s}+\log \left(\log \gamma_{s}\right)+p_{s}^{\prime}\right. \\
& \left.\quad+\sum_{k \in \mathcal{N} \backslash\{s\}} p_{k}^{\prime \prime}-x_{s}^{\prime}\right) \\
& \quad+\sum_{s \in \mathcal{N}} \mu_{s}\left(1-y_{s}-k_{p} \gamma_{s}^{-\left(M_{T}-r_{s}\right)\left(M_{R}-r_{s}\right)}\right)
\end{aligned}
$$

The Lagrange dual function is:

$$
\Phi(\boldsymbol{\lambda}, \boldsymbol{\mu})=\max _{\mathbf{x}^{\prime}, \mathbf{y}, \mathbf{r}, \mathbf{p}^{\prime}, \mathbf{p}^{\prime \prime}} L\left(\mathbf{x}^{\prime}, \mathbf{y}, \mathbf{r}, \mathbf{p}^{\prime}, \mathbf{p}^{\prime \prime}, \boldsymbol{\lambda}, \boldsymbol{\mu}\right)
$$

where $\mathbf{x}^{\prime}, \mathbf{y}, \mathbf{r}, \mathbf{p}^{\prime}, \mathbf{p}^{\prime \prime}$ are subject to the third to the ninth constraints of (25).

The dual optimization problem can be formulated as:

$$
\min \Phi(\boldsymbol{\lambda}, \boldsymbol{\mu}), \quad \text { subject to } \boldsymbol{\lambda} \succeq \mathbf{0}, \quad \text { and } \boldsymbol{\mu} \succeq \mathbf{0},
$$

which is optimized over $\boldsymbol{\lambda}$ and $\boldsymbol{\mu}$.
Due to the separability of variables in (28), the problem can be decomposed into two sub-problems. The maximization of the Lagrangian over $\mathbf{x}^{\prime}, \mathbf{y}, \mathbf{r}, \mathbf{p}^{\prime}, \mathbf{p}^{\prime \prime}$ can be conducted in parallel at the application layer for the target throughput $\mathbf{x}^{\prime}$ and reliability $\mathbf{y}$ :

$$
\begin{array}{cll}
\max & \sum_{i \in \mathcal{N}_{1}} \beta U_{1}^{\prime}\left(x_{i}^{\prime}, y_{i}\right)+\sum_{j \in \mathcal{N}_{2}}(1-\beta) U_{2}\left(y_{j}\right) \\
& -\sum_{s \in \mathcal{N}}\left(\lambda_{s} x_{s}^{\prime}+\mu_{s} y_{s}\right) & \\
\text { subject to } & \log \check{x}_{1} \leq x_{i}^{\prime} \leq \log \hat{x}, & \forall i \in \mathcal{N}_{1} \\
& \log R \leq x_{j}^{\prime} \leq \log \hat{x}, & \forall j \in \mathcal{N}_{2} \\
& \check{y}_{1} \leq y_{i} \leq 1, & \forall i \in \mathcal{N}_{1} \\
& Q \leq y_{j} \leq 1, & \forall j \in \mathcal{N}_{2}
\end{array}
$$

which is optimized over $\mathbf{x}^{\prime}$ and $\mathbf{y}$.

And, on the MAC/PHY layers for the transmission probability $\mathbf{p}$ and MIMO diversity gain $\mathbf{r}$ :

$$
\begin{array}{cll}
\max & \sum_{s \in \mathcal{N}}\left[\lambda_{s}\left(\log r_{s}+p_{s}^{\prime}+\sum_{k \in \mathcal{N} \backslash\{s\}} p_{k}^{\prime \prime}\right)\right. \\
& \left.-\mu_{s} k_{p} \gamma_{s}^{-\left(M_{T}-r_{s}\right)\left(M_{R}-r_{s}\right)}\right] & \\
\text { subject to } & e^{p_{s}^{\prime}}+e^{p_{s}^{\prime \prime}} \leq 1, & \forall s \in \mathcal{N} \\
& p_{s}^{\prime}, p_{s}^{\prime \prime} \leq 0, & \forall s \in \mathcal{N} \\
& 0 \leq r_{s} \leq \Delta, & \forall s \in \mathcal{N}
\end{array}
$$

which is optimized over $\mathbf{p}$ and $\mathbf{r}$.

The above problem can be separately solved at each wireless station locally. For $i \in \mathcal{N}_{1}$ with $A C_{1}$ traffic, it solves the following problem to obtain its target $x_{i}^{\prime}$ and $y_{i}$ values:

$$
\begin{array}{cl}
\max & \beta U_{1}^{\prime}\left(x_{i}^{\prime}, y_{i}\right)-\left(\lambda_{i} x_{i}^{\prime}+\mu_{i} y_{i}\right) \\
\text { subject to } & \log \check{x}_{1} \leq x_{i}^{\prime} \leq \log \hat{x}, \\
& \check{y}_{1} \leq y_{i} \leq 1,
\end{array}
$$

which is optimized over $x_{i}^{\prime}$ and $y_{i}$.

For station $j \in \mathcal{N}_{2}$ with $A C_{2}$ traffic, its target $x_{j}^{\prime}$ and $y_{j}$ values can be calculated from:

$$
\begin{array}{cl}
\max & (1-\beta) U_{2}\left(y_{j}\right)-\left(\lambda_{j} x_{j}^{\prime}+\mu_{j} y_{j}\right) \\
\text { subject to } & \log R \leq x_{j}^{\prime} \leq \log \hat{x} \\
& Q \leq y_{i} \leq 1
\end{array}
$$

which is optimized over $x_{j}^{\prime}$ and $y_{j}$.

For station $s \in \mathcal{N}$, the MIMO diversity gain $\mathbf{r}$ at the PHY layer can be calculated from:

$$
\begin{array}{cl}
\max & \lambda_{s} \log r_{s}-\mu_{s} k_{p} \gamma_{s}^{-\left(M_{T}-r_{s}\right)\left(M_{R}-r_{s}\right)} \\
\text { subject to } & 0 \leq r_{s} \leq \Delta .
\end{array}
$$

From (32), since $\sum_{s \in \mathcal{N}} \lambda_{s}\left(p_{s}^{\prime}+\sum_{k \in \mathcal{N} \backslash\{s\}} p_{k}^{\prime \prime}\right)=$ $\sum_{s \in \mathcal{N}}\left(\lambda_{s} p_{s}^{\prime}+\sum_{k \in \mathcal{N} \backslash\{s\}} \lambda_{k} p_{s}^{\prime \prime}\right)$, the transmission probability $\mathbf{p}$ can be solved at each wireless station $s$ by:

$$
\begin{array}{cl}
\max & \lambda_{s} p_{s}^{\prime}+\sum_{k \in \mathcal{N} \backslash\{s\}} \lambda_{k} p_{s}^{\prime \prime} \\
\text { subject to } & e^{p_{s}^{\prime}}+e^{p_{s}^{\prime \prime}} \leq 1, \\
& p_{s}^{\prime}, p_{s}^{\prime \prime} \leq 0 .
\end{array}
$$


With the subgradient projection algorithm, $\lambda_{s}$ and $\mu_{s}$ can be updated at each station as follows:

$$
\begin{aligned}
\lambda_{s}(t+1)= & {\left[\lambda_{s}(t)-\delta(t)\left(\log r_{s}+p_{s}^{\prime}+\sum_{k \in \mathcal{N} \backslash\{s\}} p_{k}^{\prime \prime}\right.\right.} \\
& \left.\left.+\log k_{c}+\log \log \gamma_{s}-x_{s}^{\prime}\right)\right]^{+} \\
\mu_{s}(t+1)= & {\left[\mu_{s}(t)-\delta(t)\left(1-y_{s}\right.\right.} \\
& \left.\left.-k_{p} \gamma_{s}^{-\left(M_{T}-r_{s}\right)\left(M_{R}-r_{s}\right)}\right)\right]^{+}
\end{aligned}
$$

where $[x]^{+}=\max (x, 0)$, and $\delta(t)$ is the diminishing step size [28] (e.g., $\delta(t)=1 /(1+t)$ ). By taking the advantage of the broadcast nature of the wireless transmission, the values of $\boldsymbol{\lambda}$ and $\boldsymbol{p}$ which are required in each iteration can be piggybacked over a broadcast frame by each station. Thus, it introduces limited overhead in the network. Another advantage of the distributed solution is that the computation complexity is reduced compared with the centralized scheme. This will benefit a mobile node which would save its CPU utilization and battery consumption. Furthermore, when the framework is extended into multiple hops, the distributed solution would introduce more substantial performance gains than the central computation. Extension of the single-hop WLAN to multiple hops is under our further study. The distributed algorithm (33) to (38) runs at each wireless station $s$ by calculating its target throughput $x_{s}$, reliability requirement $y_{s}$, MIMO diversity gain $r_{s}$, and transmission probability $p_{s}$. The $r_{s}$ and $p_{s}$ results can then be used to adjust the PHY/MAC operation parameters.

\section{NUM-S: Utility-Maximization with Separated PHY/MAC Layers}

For performance analysis, we propose a simplified scheme in which the persistent probabilities at the MAC layer and the multiplexing-diversity tradeoff scheme at the physical layer are determined separately. We call this scheme $N U M-S$, and use it as a baseline for performance comparison in Section IV.

At the MAC layer, it is shown that under identical traffic and data rate, the network throughput is maximized with each node transmitting with persistent probability [29]:

$$
p_{s}=1 / N, \quad s \in \mathcal{N},
$$

where $N=|\mathcal{N}|$. If $p_{s}$ is selected from (39), then the MIMO multiplexing-diversity tradeoff at the physical layer for each station becomes independent of each other and can be computed as follows: At the physical layer, the $A C_{2}$ station $j$ can calculate its required level of MIMO multiplexing gain for providing the CBR data rate $R$ from (18):

$$
r_{j}=\frac{R N^{N}}{k_{c} \log \gamma_{j}(N-1)^{N-1}}, \quad j \in \mathcal{N}_{2} .
$$

The value $r_{j}$ is the minimum multiplexing gain required for sustaining the data rate $R$, which leads to the corresponding maximum diversity gain from (2) because $d_{j}$ is a strictly decreasing function of $r_{j}$. This maximum diversity gain $d_{j}$ in turn provides the maximum reliability $y_{j}$ available for station $j$ as from (5). Because the utility function (22) is strictly increasing with $y_{j}$, this multiplexing-diversity tradeoff maximizes the utility for $A C_{2}$ stations.
TABLE II

Simulation PARAMETERS

\begin{tabular}{|l|c|}
\hline \hline Basic Rate & $10 \mathrm{Mbps}$ \\
\hline Maximum Data Rate & $80 \mathrm{Mbps}$ \\
\hline DSSS PLCP Preamble and Header & $192 \mathrm{bits}$ \\
\hline MAC Header & $192 \mathrm{bits}$ \\
\hline FCS (Frame Check Sequence) & 32 bits \\
\hline ACK Frame Size (include Headers) & 304 bits \\
\hline Data Packet Size & 1000 bytes \\
\hline Time Slot & $9 \mu \mathrm{s}$ \\
\hline SIFS & $10 \mu \mathrm{s}$ \\
\hline AIFS & $28 \mu \mathrm{s}$ \\
\hline Maximum Number of Retransmissions & 5 \\
\hline \hline
\end{tabular}

For the $A C_{1}$ stations, maximizing $U_{1}(x y)$ in (27) is equivalent to maximizing $x y$ because it is strictly increasing with $x y$. We can solve the optimal multiplexing gain $r_{i}$ for each $A C_{1}$ station $i \in \mathcal{N}_{1}$ with the following simple constrained maximization problem:

$$
\begin{array}{cl}
\max _{r_{i}} & k_{c} r_{i} \log \left(\gamma_{i}\right) \frac{(N-1)^{N-1}}{N^{N}}\left(1-k_{p} \gamma_{i}^{-d\left(r_{i}\right)}\right), \\
\text { subject to } & \check{x}_{1} \leq k_{c} r_{i} \log \left(\gamma_{i}\right) \frac{(N-1)^{N-1}}{N^{N}} \leq \hat{x}, \\
& \check{y}_{1} \leq 1-k_{p} \gamma_{s}^{-d\left(r_{i}\right)} \leq 1, \\
& d\left(r_{i}\right)=\left(M_{T}-r_{i}\right)\left(M_{R}-r_{i}\right), \\
& 0 \leq r_{i} \leq \min \left(M_{T}, M_{R}\right) .
\end{array}
$$

The solution of problem (41) provides each $A C_{1}$ station with its MIMO multiplexing gain $r_{i}$, and the transmission reliability $y_{i}$ can be subsequently calculated from (5). With the reliability results from $A C_{2}$ stations, we can obtain the network utility from (25) to compare with the NUM-D scheme.

\section{Performance Evaluation}

\section{A. Results for 802.11e EDCA}

We carry out network simulations by using the ns-2 [30] simulator to study the effectiveness of the cross-layer optimization framework proposed in this paper. The simulation parameters are shown in Table II. We consider a WLAN with 10 wireless stations. Five of them have $A C_{1}$ traffic, and five of them have $A C_{2}$ traffic. To effectively study the traffic differentiation effects on our proposed schemes, we use the same SNR values for each pair of $A C_{1}$ and $A C_{2}$ stations. That is $\gamma_{i}=\gamma_{i+5}$ (for $i=1, \ldots, 5$ ). Each $\gamma_{i}$ is a Gaussian distributed random variable with a mean value of $\gamma_{m}=10 \mathrm{~dB}$, and a variance of $\gamma_{v}=5$. To avoid obtaining a too low SNR from the Gaussian random number generator, which will likely lead to no feasible solution for the optimization problem, we set a lower SNR bound of $3 \mathrm{~dB}$. This setup tries to simulate a WLAN with wireless stations randomly located within certain distances from the AP. Other assumptions such as uniformly distributed SNRs have also been tested, and do not seem to have a major impact on the performance comparisons. In fact, the $U-M A C$ and $D-M A C$ models do not make any assumptions on the SNR's distributions. And our tests also show that similar performance trends are observed under differently simulated SNRs. Thus, only results from the Gaussian distributed SNRs are presented. 


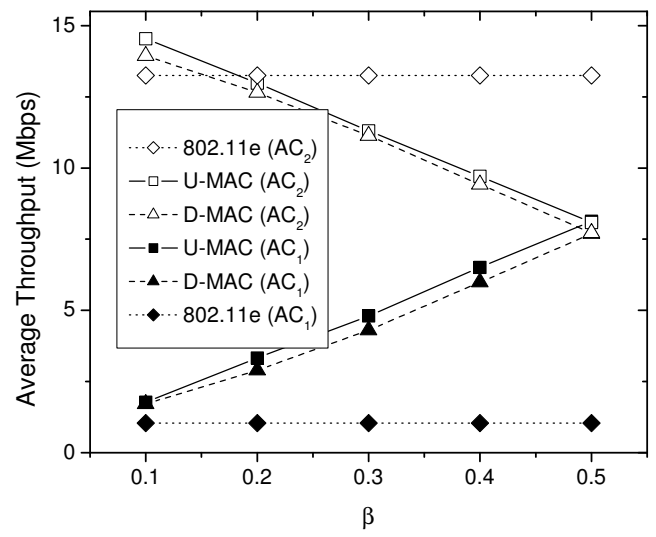

(a) Average throughput performance between U-MAC, DMAC, and 802.11e.

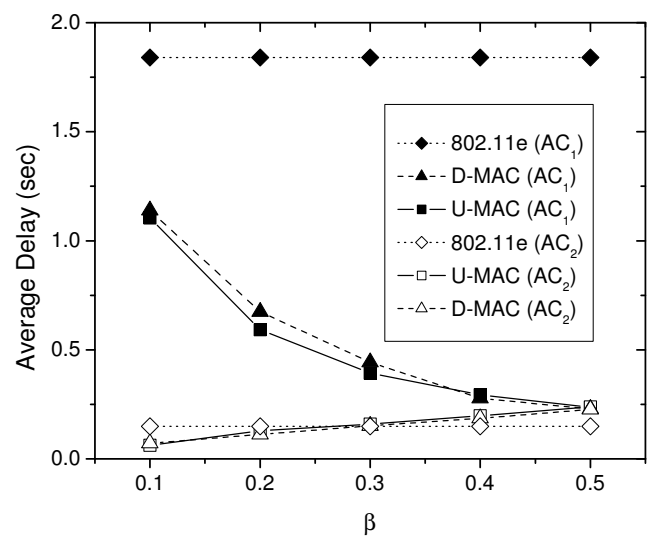

(b) Average delay performance between U-MAC, D-MAC, and 802.11 e.

Fig. 1. Performance of U-MAC and D-MAC versus $\beta$.

For $U-M A C$ and $D-M A C$, the following parameters are used: $k_{c}=20 \mathrm{MHz}, k_{p}=0.15, M_{T}=2, M_{R}=3, \breve{W}_{s}=\check{W}_{a}=7$, $\hat{W}_{s}=\hat{\bar{W}}_{a}=1023, \alpha=1.1, \check{y}_{1}=0.7, \check{y}_{2}=0.85$, and $\Gamma=1$. With a maximum of five retransmissions, the maximum contention window size is set to be $2^{5}=32$ times the minimum contention window size. The resulting MIMO multiplexing gain $r$ and contention window size $C W$ from the solution of (16) or (17) are used in the ns-2 simulator to assign the proper physical and MAC operating parameters. We vary $\beta$ from 0.1 to 0.5 to study the effectiveness of the traffic differentiation effect of our proposed schemes. Smaller $\beta$ improves the higher priority $A C_{2}$ 's performance, but at a price of decreasing performance of $A C_{1}$. A desired tradeoff may be obtained by assigning the appropriate $\beta$ by the network administrator.

We compare the proposed adaptive cross-layer schemes $U$ $M A C$ and D-MAC with the original 802.11e MAC. We look at the following three main performance metrics: the traffic's throughput, delay and the network's utility. The throughput is calculated as the end-to-end network throughput above the MAC layer, which is the number of bits transmitted in the transport layer protocol data unit (PDU) divided by time. For the original 802.11e MAC, the $\bar{W}_{1}$ and $\bar{W}_{2}$ are chosen as 7 and 31 , respectively, according to the standard specifications. In order to have a meaningful performance comparison, we

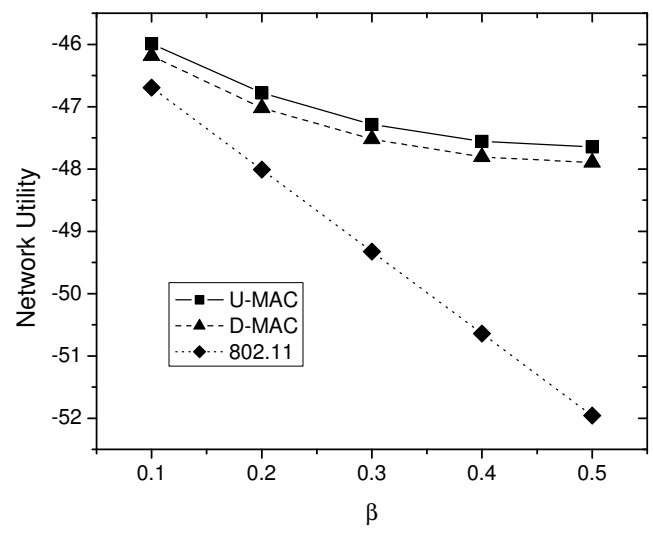

Fig. 2. Network utility of U-MAC and D-MAC versus $\beta$.

also use MIMO links with the 802.11e MAC. The multiplexing gain $r_{s}$ of wireless station $s$ is chosen without the cross-layer optimization as done in the $U-M A C$ and $D-M A C$ schemes. Its $r_{s}$ is chosen by (5) such that wireless station $s$ would achieve a link reliability of 0.9 with its channel SNR $\gamma_{s}$. We will study the effectiveness of the tuning $\beta$ on the traffic QoS differentiation. The network total utility is used to evaluate the effectiveness of the schemes in achieving better utility values, which corresponds to a higher network revenue and better user QoS satisfactions.

Fig. 1(a) shows the aggregated throughput of each AC. Because of the static nature of the 802.11e MAC scheme, its performance values do not change with $\beta$, and are drawn as a horizontal line in the figure to be used as a comparison baseline. The medium access delay is shown in Fig. 1(b). An increase in $\beta$ decreases the priority differentiation between the two ACs. When $\beta$ reaches $0.5, A C_{1}$ and $A C_{2}$ reach equal priority and have comparable performances.

The network utility is shown in Fig. 2. Although the 802.11e MAC does not consider $\beta$, its $x_{s}, y_{s}$ values are also substituted into (15) to calculate the network utility under different $\beta$. Fig. 2 shows that the $U-M A C$ and $D-M A C$ maintain a relatively high network utility, while the $802.11 \mathrm{e}$ is not adaptive to different $\beta$ adjustments and its network utility decreases sharply when the utility function demands less traffic differentiation by increasing the $\beta$ value.

Simulation results show that the adaptive $U-M A C$ and $D$ $M A C$ schemes have good flexibility in adapting to the network's different assignment of weights to the two access categories. They also consistently outperform the original $802.11 \mathrm{e}$ MAC in every performance measure because they jointly adapt the minimum contention window size values and the MIMO coding schemes. These results verify that those simplifying assumptions that we made to formulate the computationally efficient NUM problem in Section II are reasonable and sound.

We can also observe that the $U$-MAC slightly outperforms the $D-M A C$, even though $D-M A C$ allows the extra flexibility of letting each station have its own $W_{s}$ parameter. The cause for this is from the coarse approximation we made in the throughput model for $D-M A C$, while the $U$-MAC model is more accurate. However, an insight that we can gain from these simulation experiments is that restricting each access 
TABLE III

NUM SOLUTION FOR THE U-MAC SCHEME.

\begin{tabular}{|c|c|c|c|c|c|c|c|c|c|c|c|}
\hline \multirow{2}{*}{\multicolumn{2}{|c|}{ Station $s$}} & \multicolumn{5}{|c|}{$A C_{1}$} & \multicolumn{5}{|c|}{$A C_{2}$} \\
\hline & & 1 & 2 & 3 & 4 & 5 & 6 & 7 & 8 & 9 & 10 \\
\hline$\beta$ & $\gamma_{s}$ & $6 \mathrm{~dB}$ & $7 \mathrm{~dB}$ & $8 \mathrm{~dB}$ & $9 \mathrm{~dB}$ & $10 \mathrm{~dB}$ & $6 \mathrm{~dB}$ & $7 \mathrm{~dB}$ & $8 \mathrm{~dB}$ & $9 \mathrm{~dB}$ & $10 \mathrm{~dB}$ \\
\hline 0.1 & $W_{s}$ & \multicolumn{5}{|c|}{220} & \multicolumn{5}{|c|}{29} \\
\hline$\overline{0.2}$ & $\overline{W_{s}}$ & \multicolumn{5}{|c|}{120} & \multicolumn{5}{|c|}{33} \\
\hline$\overline{0.3}$ & $W_{s}$ & \multicolumn{5}{|c|}{84} & \multicolumn{5}{|c|}{38} \\
\hline 0.4 & $W_{s}$ & \multicolumn{5}{|c|}{65} & \multicolumn{5}{|c|}{45} \\
\hline 0.5 & $\overline{W_{s}}$ & \multicolumn{5}{|c|}{53} & \multicolumn{5}{|c|}{53} \\
\hline 0.1 & $r_{s}$ & 1.552 & 1.522 & 1.496 & 1.470 & 1.442 & 1.549 & 1.528 & 1.502 & 1.476 & 1.449 \\
\hline$\overline{0.2}$ & $r_{s}$ & 1.554 & 1.524 & 1.497 & 1.471 & 1.444 & 1.549 & 1.528 & 1.501 & 1.475 & 1.449 \\
\hline$\overline{0.3}$ & $r_{s}$ & 1.555 & 1.525 & 1.498 & 1.472 & 1.445 & 1.549 & 1.528 & 1.501 & 1.475 & 1.448 \\
\hline 0.4 & $r_{s}$ & 1.556 & 1.526 & 1.499 & 1.473 & 1.446 & 1.549 & 1.527 & 1.500 & 1.474 & 1.447 \\
\hline 0.5 & $r_{s}$ & 1.557 & 1.527 & 1.500 & 1.474 & 1.447 & 1.549 & 1.526 & 1.500 & 1.474 & 1.447 \\
\hline
\end{tabular}

TABLE IV

NUM SOLUTION FOR THE D-MAC SCHEME.

\begin{tabular}{|c|c|c|c|c|c|c|c|c|c|c|c|}
\hline \hline & \multicolumn{9}{|c|}{$A C_{1}$} & \multicolumn{6}{|c|}{$A C_{2}$} \\
\hline \multicolumn{2}{|c|}{ Station $s$} & 1 & 2 & 3 & 4 & 5 & 6 & 7 & 8 & 9 & 10 \\
\hline$\beta$ & $\gamma_{s}$ & $6 \mathrm{~dB}$ & $7 \mathrm{~dB}$ & $8 \mathrm{~dB}$ & $9 \mathrm{~dB}$ & $10 \mathrm{~dB}$ & $6 \mathrm{~dB}$ & $7 \mathrm{~dB}$ & $8 \mathrm{~dB}$ & $9 \mathrm{~dB}$ & $10 \mathrm{~dB}$ \\
\hline 0.1 & $W_{s}$ & 36 & 33 & 31 & 29 & 27 & 7 & 7 & 7 & 7 & 7 \\
\hline 0.2 & $W_{s}$ & 23 & 22 & 20 & 19 & 18 & 7 & 7 & 7 & 7 & 7 \\
\hline 0.3 & $W_{s}$ & 17 & 16 & 15 & 14 & 13 & 8 & 8 & 7 & 7 & 7 \\
\hline 0.4 & $W_{s}$ & 14 & 13 & 12 & 11 & 10 & 10 & 9 & 8 & 8 & 7 \\
\hline 0.5 & $W_{s}$ & 11 & 10 & 10 & 9 & 9 & 11 & 10 & 10 & 9 & 9 \\
\hline 0.1 & $r_{s}$ & 1.632 & 1.631 & 1.632 & 1.636 & 1.640 & 1.539 & 1.528 & 1.523 & 1.521 & 1.522 \\
\hline 0.2 & $r_{s}$ & 1.562 & 1.569 & 1.576 & 1.583 & 1.591 & 1.538 & 1.540 & 1.533 & 1.531 & 1.531 \\
\hline 0.3 & $r_{s}$ & 1.538 & 1.546 & 1.554 & 1.563 & 1.571 & 1.528 & 1.536 & 1.545 & 1.547 & 1.546 \\
\hline 0.4 & $r_{s}$ & 1.530 & 1.539 & 1.548 & 1.557 & 1.565 & 1.526 & 1.535 & 1.544 & 1.553 & 1.561 \\
\hline 0.5 & $r_{s}$ & 1.528 & 1.537 & 1.546 & 1.554 & 1.563 & 1.528 & 1.537 & 1.546 & 1.554 & 1.563 \\
\hline \hline
\end{tabular}

category to have uniform minimum contention window sizes may be sufficient to provide good performance gain in the MIMO-enabled cross layer design framework. We may be able to improve the performance of $D-M A C$ by further refining its throughput model.

To have a better understanding of the system behavior near the optimal values, we present a set of results of the system variables from the NUM framework in Tables III and IV. Ten wireless stations are also used in the tests. The link SNR values $\gamma_{i}=\gamma_{i+5}$ (for $i=1, \ldots, 5$ ) are set at increasing values (from $6 \mathrm{~dB}$ to $10 \mathrm{~dB}$ ) instead of random numbers so that it is easier to observe the behavior of the system variables. $\beta$ is increased from 0.1 to 0.5 . From the tables, we can see that the difference of the diversity gain and contention window size of the two $A C$ 's stations diminishes when $\beta$ approaches 0.5. In previous simulation tests, the $D-M A C$ slightly under-performs $U-M A C$. From these two tables, we can see that the $D-M A C$ tends to use a smaller contention window size than the $U$-MAC model, which is likely the cause for the performance loss. A more accurate throughput model for $D-M A C$ will be needed for improving this performance gap.

\section{B. Results for Slotted Aloha MAC}

The slotted Aloha schemes NUM-D and NUM-S are studied with numerical tests in Matlab. The following parameters are used: $\check{x}=0.01 \mathrm{Mbps}, \hat{\gamma}=30 \mathrm{~dB}, Q=0.85$, and the other parameters use the same values as in Section IV-A. The stopping criteria for $N U M-D$ is that $\left|\lambda_{s}(t+1)-\lambda_{s}(t)\right|$ and $\left|\mu_{s}(t+1)-\mu_{s}(t)\right|$ are less than $10^{-6}$ for all $s$.

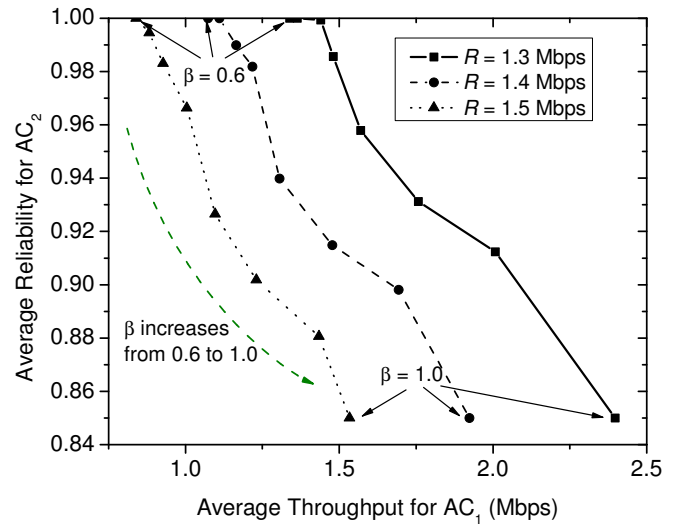

Fig. 3. The tradeoff between throughput and reliability for slotted Aloha.

1) Effect of $\beta$ on the Network Utility Function: First, we examine the effectiveness of the network utility function (23) in determining the tradeoff between the throughput of $A C_{1}$ and the reliability of $A C_{2}$. We vary the parameter $\beta$ from 0.6 to 1 with a step size of 0.05 , and solve problem (25). We use the Gaussian distributed random variable with a mean value of $\gamma_{m}$ and a variance of $\gamma_{v}$ to generate the SNR values in simulations. The results for $N_{1}=N_{2}=5$, $\gamma_{m}=8 d B, \gamma_{v}=0.5 \gamma_{m}$, and varying $R$ are shown in Fig. 3. The throughput is calculated from (18) which is the throughput for transmitting the MAC layer protocol data unit with MAC frame headers. We can see that for a smaller $\beta$, the throughput in $A C_{1}$ is given lower priority, but the network achieves higher reliability for the $A C_{2}$ traffic. As $\beta$ 


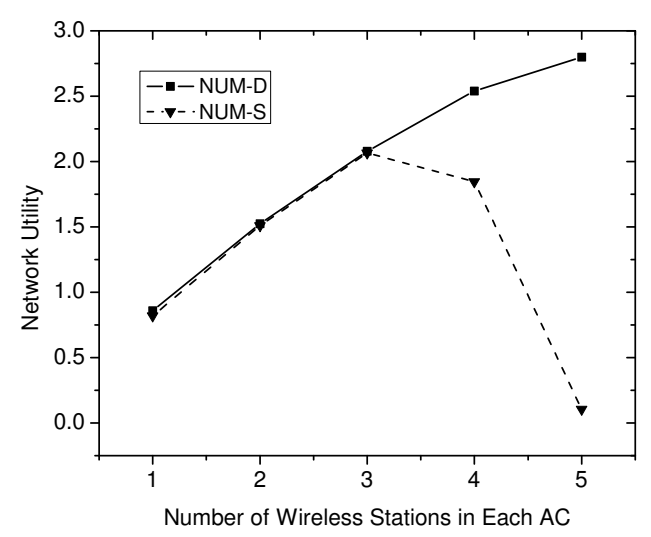

(a) Network utility versus the number of wireless stations.

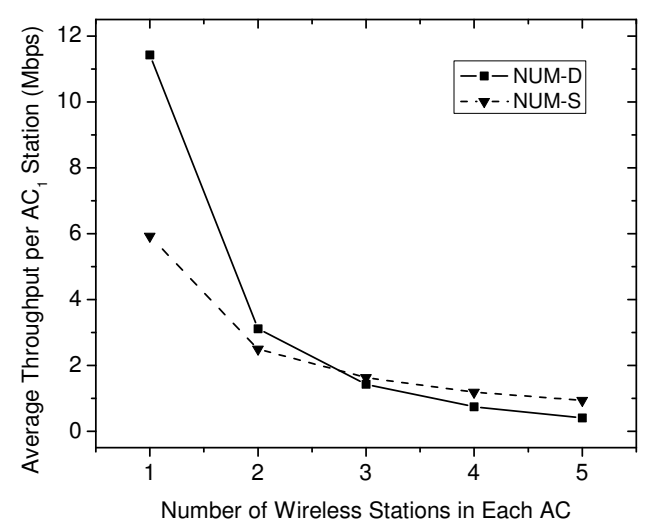

(b) Average throughput performance for best-effort traffic.

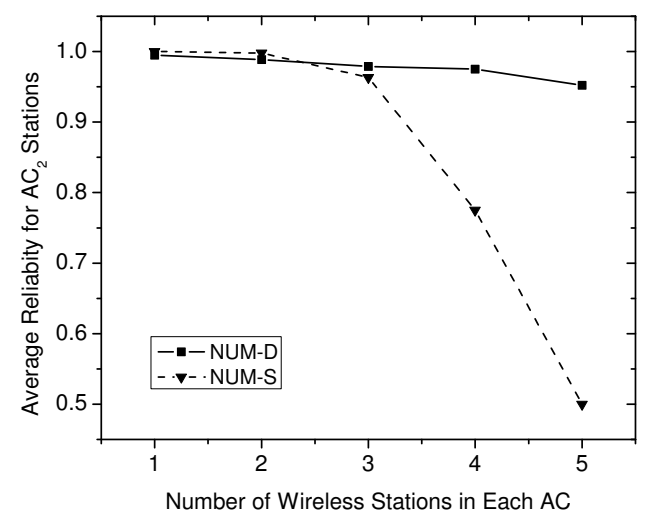

(c) Average reliability for real-time traffic.

Fig. 4. Slotted Aloha under NUM-D and NUM-S with different number of wireless stations.

increases, the throughput in $A C_{1}$ significantly increases at the price of decreasing reliability on $A C_{2}$ traffic. The area below each curve is effectively the achievable throughput-reliability region for a specified $R$. When $R$ increases, this region shrinks because more resources have to be allocated to guarantee the higher throughput requirement from the $A C_{2}$ traffic. Fig. 3 shows that the utility function (23) has great flexibility in adapting different user QoS requirements by adjusting the parameter $\beta$. If the network operators can generate higher revenues by providing high reliability for $A C_{2}$ traffic, they may tend to choose a smaller $\beta$ value. In the following tests, we choose $\beta=0.85$.

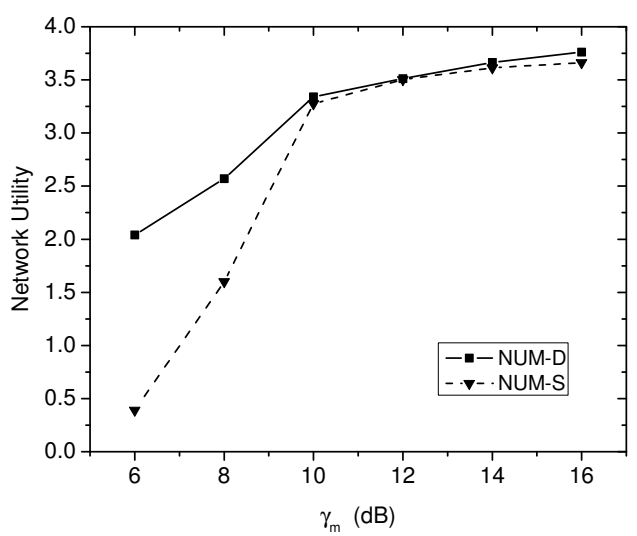

(a) Network utility versus the average SNR.

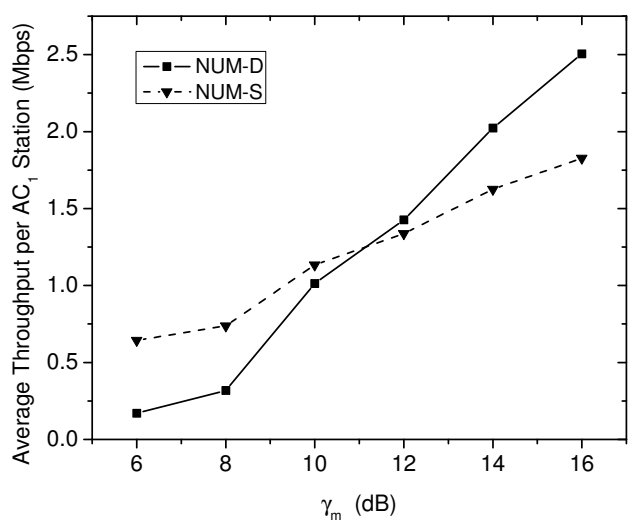

(b) Average throughput performance for best-effort traffic.

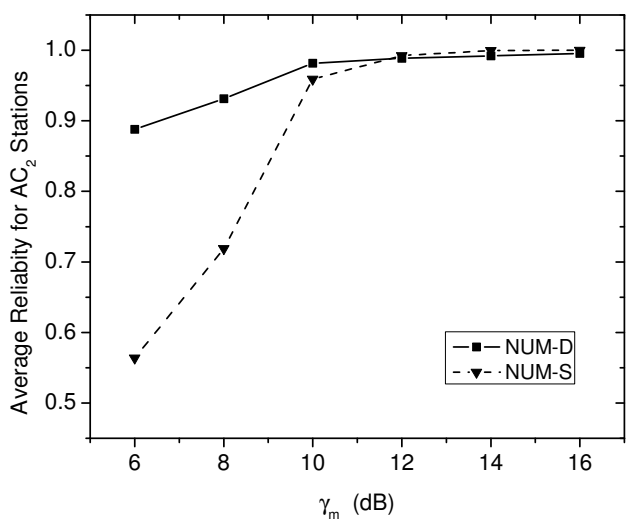

(c) Average reliability for real-time traffic.

Fig. 5. Slotted Aloha under NUM-D and NUM-S with different SNR.

2) Performance under Different Number of Stations: In this experiment, $\gamma_{m}$ is fixed at $8 \mathrm{~dB}$, and $\gamma_{v}=0.5 \gamma_{m}$. We vary the number of stations in each $\mathrm{AC}$ from 1 to 5 . $R$ is set at $1.5 \mathrm{Mbps}$. The resulting network utility, the throughput $A C_{1}$ and reliability performance in $A C_{2}$ are shown in Figs. 4(a) to 4(c). When the number of stations is small, the NUM-D scheme achieves much higher $A C_{1}$ throughput than NUM-S. When the number of stations increases, the performance gain on $A C_{1}$ 's throughput by $N U M-D$ diminishes. But $N U M-D$ is able to maintain the $A C_{2}$ 's reliability above $95 \%$, while $N U M$ $S$ 's reliability performance significantly deteriorates. Overall, the network utility achieved by $N U M-D$ is consistently higher 
TABLE V

SOLUTION FOR THE NUM-D SCHEME.

\begin{tabular}{|c|c|c|c|c|c|c|c|c|c|c|}
\hline \hline & \multicolumn{9}{|c|}{$A C_{1}$} & \multicolumn{6}{|c|}{$A C_{2}$} \\
\hline Station $s$ & 1 & 2 & 3 & 4 & 5 & 6 & 7 & 8 & 9 & 10 \\
\hline$\gamma_{s}$ & $6 \mathrm{~dB}$ & $7 \mathrm{~dB}$ & $8 \mathrm{~dB}$ & $9 \mathrm{~dB}$ & $10 \mathrm{~dB}$ & $6 \mathrm{~dB}$ & $7 \mathrm{~dB}$ & $8 \mathrm{~dB}$ & $9 \mathrm{~dB}$ & $10 \mathrm{~dB}$ \\
\hline$p_{s}$ & 0.090 & 0.089 & 0.089 & 0.088 & 0.087 & 0.138 & 0.122 & 0.109 & 0.099 & 0.090 \\
\hline$r_{s}$ & 1.562 & 1.575 & 1.587 & 1.599 & 1.610 & 1.124 & 1.149 & 1.173 & 1.195 & 1.216 \\
\hline$x_{s}(\mathrm{Mbps})$ & 0.864 & 0.969 & 1.074 & 1.179 & 1.283 & 1.000 & 1.000 & 1.000 & 1.000 & 1.000 \\
\hline$y_{s}$ & 0.843 & 0.857 & 0.869 & 0.879 & 0.888 & 0.973 & 0.980 & 0.982 & 0.986 & 0.989 \\
\hline$\lambda_{s}$ & 0.085 & 0.085 & 0.084 & 0.083 & 0.082 & 0.130 & 0.115 & 0.103 & 0.093 & 0.085 \\
\hline$\mu_{s}$ & 0.102 & 0.099 & 0.097 & 0.094 & 0.092 & 0.943 & 0.937 & 0.934 & 0.930 & 0.927 \\
\hline \hline
\end{tabular}

than NUM-S.

3) Performance under Different SNRs: In this experiment, $N_{1}$ and $N_{2}$ are set to 5 . The network average $\gamma_{m}$ varies from $6 \mathrm{~dB}$ to $16 \mathrm{~dB}, \gamma_{v}=0.5 \gamma_{m}$. $R$ is equal to $1.0 \mathrm{Mbps}$. Figs. 5(a) to 5(c) show the network utility, $A C_{1}$ 's throughput and $A C_{2}$ 's reliability achieved with these two schemes. Results show that in the lower SNR region, NUM-D sacrifices a portion of $A C_{1}$ 's throughput to achieve consistently high reliability for $A C_{2}$, while at the higher SNR region, $N U M$ $D$ achieves significantly higher throughput than $N U M-S$ and also maintains $A C_{2}$ 's reliability greater than $99 \%$. As a result, it successfully achieves a good balance between throughput for best-effort traffic and reliability for real-time traffic and attains higher network utility than the NUM-S scheme.

To study the detailed system variable behavior at the optimal solution, we also present a set of results with pre-determined SNR values in Table V. We can see that the solutions provide the required $1 \mathrm{Mbps}$ throughput for the $A C_{2}$ traffic with good reliability. The best-effort $A C_{1}$ traffic tends to use higher a multiplexing gain $r_{s}$ to achieve a higher data rate. The stations with higher SNR links can use a higher multiplexing gain and lower transmission probability to achieve the required link reliability and throughput.

The above results show that the cross-layer network utility maximization formulation of (24) successfully balances between the QoS requirements of the two access categories by jointly adapting MAC and MIMO physical layer parameters.

\section{Conclusions}

This paper proposed a MAC/PHY cross-layer design with network utility maximization in a WLAN with multiple classes of traffic. Two types of MAC protocol are considered. For the CSMA/CA-based 802.11e EDCA MAC, the minimum contention window size at the MAC layer is jointly optimized with the multiplexing-diversity gain tradeoff at the physical layer with MIMO antennas. The utility-based crosslayer design is shown to be flexible in adjusting the system performance in regard to QoS tradeoff for different access categories of traffic. We further analyzed the slotted Aloha MAC using the NUM approach as well, and were able to derive distributed solutions with variable transformation and dual decomposition of the original NUM problem. Simulation results are presented for both MAC designs, which show the effectiveness in system performance improvement. Future work will consider extending the existing framework to a multi-hop scenario and further extending the CSMA/CA MAC to work in a distributed manner.

\section{APPENDIX}

A. Proof of the concavity of the utility function in (25)

For $\alpha>1$, we define the constants $C_{1}$ and $C_{2}$ as follows:

$$
\begin{aligned}
C_{1} & =\frac{1}{(\hat{x})^{1-\alpha}-\left(\check{x}_{1} \check{y}_{1}\right)^{1-\alpha}} \\
C_{2} & =\frac{1}{1-Q^{1-\alpha}}
\end{aligned}
$$

It is easy to see that $C_{1}<0$ and $C_{2}<0$, because $t^{1-\alpha}$ is a strictly decreasing function of $t$ for $\alpha>1$. As a result, $C_{2} y^{1-\alpha}$ is a concave function of $y$ for $\alpha>1$. Thus, $U_{2}(y)$ is a concave function.

For $U_{1}^{\prime}\left(x^{\prime}, y\right)$, the Hessian matrix of $C_{1}\left(e^{x^{\prime}} y\right)^{1-\alpha}$ is:

$$
\begin{aligned}
\mathbf{H}= & \nabla^{2} C_{1}\left(e^{x^{\prime}} y\right)^{1-\alpha}=C_{1} e^{(1-\alpha) x^{\prime}} \\
& \times\left[\begin{array}{ll}
(1-\alpha)^{2} y^{1-\alpha} & (1-\alpha)^{2} y^{-\alpha} \\
(1-\alpha)^{2} y^{-\alpha} & -\alpha(1-\alpha) y^{-\alpha-1}
\end{array}\right]
\end{aligned}
$$

For any non-zero vector $\mathbf{z}=\left[\begin{array}{ll}z_{1} & z_{2}\end{array}\right]$ and $\alpha>1$, we have:

$$
\begin{aligned}
\mathbf{z H z} \mathbf{z}^{T}= & C_{1}(1-\alpha) e^{(1-\alpha) x^{\prime}} y^{-\alpha-1}\left[(1-\alpha)\left(y z_{1}+z_{2}\right)^{2}\right. \\
& \left.-z_{2}^{2}\right]<0
\end{aligned}
$$

which shows that $\mathbf{H}$ is negative definite. As a result, the utility function $U_{1}^{\prime}\left(x^{\prime}, y\right)$ is a concave function of $\left\{x^{\prime}, y\right\}$.

\section{B. Proof of convexity of the constraints in (25)}

For a convex optimization problem, its equality constraints should be affine, and the function $f(x)$ in the inequality constraint $f(x) \leq 0$ should be convex.

It is easy to see that the first constraint in (25) is affine. For the second constraint, in order for the function $w\left(r_{s}\right)=$ $\gamma_{s}^{-\left(M_{T}-r_{s}\right)\left(M_{R}-r_{s}\right)}$ to be convex, it is required that:

$$
\begin{aligned}
\nabla^{2} w\left(r_{s}\right)= & \gamma_{s}^{-\left(M_{T}-r_{s}\right)\left(M_{R}-r_{s}\right)}\left[\left(M_{T}+M_{R}-2 r_{s}\right)^{2} \ln \gamma_{s}\right. \\
& -2] \ln \gamma_{s} \geq 0 .
\end{aligned}
$$

This gives an upper bound of $r_{s}$, which is reflected in (26).

The third constraint is relaxed from the equality constraint $\left(e^{p_{s}^{\prime}}+e^{p_{s}^{\prime \prime}}=1\right)$ to an inequality constraint. The main concern is that a non-linear equality constraint makes the problem nonconvex. With this relaxation to inequality constraint, the problem becomes convex. Intuitively, this relaxation is reasonable. Suppose that $e^{p_{s}^{\prime}}+e^{p_{s}^{\prime \prime}}$ is strictly less than 1 , we can increase 
$p_{s}^{\prime}$ or $p_{s}^{\prime \prime}$ to reach the upper limit 1 . An increase in $p_{s}^{\prime}$ or $p_{s}^{\prime \prime}$ will lead to relaxing the first constraint, which in turn may lead to higher $x_{s}^{\prime}$, resulting in better network utility. As a result, we may expect that this upper bound of 1 is always tight. Thus, problem (25) is a convex optimization problem over variables $\left\{\mathbf{x}^{\prime}, \mathbf{y}, \mathbf{r}, \mathbf{p}^{\prime}, \mathbf{p}^{\prime \prime}\right\}$.

\section{REFERENCES}

[1] IEEE 802.11e WG, "Wireless LAN MAC and PHY specifications amendment 8: MAC quality of service enhancements," Sept. 2005.

[2] G. Bianchi, "Performance analysis of the IEEE 802.11 distributed coordination function," IEEE J. Select. Areas Commun., vol. 18, no. 3, pp. 535-547, Mar. 2000.

[3] F. Cali, M. Conti, and E. Gregori, "Dynamic tuning of the IEEE 802.11 protocol to achieve a theoretical throughput limit," IEEE/ACM Trans. Networking, vol. 8, no. 6, pp. 785-799, Dec. 2000.

[4] IEEE 802.11n WG, “IEEE 802.11n Draft 4.0," May 2008.

[5] A. J. Paulraj, D. A. Gore, R. U. Nabar, and H. Bolcskei, "An overview of MIMO communications - a key to Gigabit wireless," Proc. of the IEEE, vol. 92, no. 2, pp. 198-218, Feb. 2004.

[6] D. Tse and P. Viswanath, Fundamentals of Wireless Communication. Cambridge University Press, 2005.

[7] M. Park, S. Choi, and S. M. Nettles, "Cross-layer MAC design for wireless networks using MIMO," in Proc. of IEEE Globecom, St. Louis, MO, Nov. 2005

[8] K. Sundaresan, R. Sivakumar, M. A. Ingram, and T. Chang, "Medium access control in ad hoc networks with MIMO links: optimization considerations and algorithms," IEEE Trans. Mobile Comput., vol. 3, no. 4, pp. 350-365, Oct.-Dec. 2004.

[9] J.-S. Park, A. Nandan, M. Gerla, and H. Lee, "SPACE-MAC: Enabling spatial reuse using MIMO channel-aware MAC," in Proc. of IEEE ICC, Seoul, Korea, May 2005.

[10] K. Sundaresan and R. Sivakumar, "Routing in ad-hoc networks with MIMO links," in Proc. of IEEE Int'l Conf. on Network Protocols, Boston, MA, Nov. 2005.

[11] A. L. Toledo and X. Wang, "TCP performance over wireless MIMO channels with ARQ and packet combining," IEEE Trans. Mobile Comput., vol. 5, no. 3, pp. 208-223, Mar. 2006.

[12] H.-F. Lu and P. Kumar, "A unified construction of space-time codes with optimal rate-diversity tradeoff," IEEE Trans. Inform. Theory, vol. 51, no. 5, pp. 1709-1730, May 2005.

[13] J. Lee, M. Chiang, and A. R. Calderbank, "Price-based distributed algorithms for rate-reliability tradeoff in network utility maximization," IEEE J. Select. Areas Commun., vol. 24, no. 5, pp. 962-976, May 2006.

[14] M. Chiang, S. H. Low, A. R. Calderbank, and J. C. Doyle, "Layering as optimization decomposition: A mathematical theory of network architectures," Proc. of the IEEE, vol. 95, no. 1, pp. 255-312, Jan. 2007.

[15] S. Choi, J. D. Prado, S. Shankar, and S. Mangold, "IEEE 802.11e contention-based channel access (EDCF) performance evaluation," in Proc. of IEEE ICC, Anchorage, Alaska, May 2003.

[16] A. Banchs, A. Azcorra, C. Garcia, and R. Cuevas, "Applications and challenges of the 802.11e EDCA mechanism: An experimental study," IEEE Network, vol. 19, no. 4, pp. 52-58, July/Aug. 2005.

[17] Y. Xiao, "Performance analysis of IEEE 802.11e EDCF under saturation condition," in Proc. of IEEE ICC, Paris, France, June 2004.

[18] Z. Kong, D. H. K. Tsang, and B. Bensaou, "Performance analysis of IEEE 802.11e contention-based channel access," IEEE J. Select. Areas Commun., vol. 22, no. 10, pp. 2095-2106, Dec. 2004.

[19] J. W. Robinson and T. S. Randhawa, "Saturation throughput analysis of IEEE 802.11e enhanced distributed coordination function," IEEE $J$. Select. Areas Commun., vol. 22, no. 5, pp. 917-928, June 2004.

[20] A. L. Toledo, T. Vercauteren, and X. Wang, "Adaptive optimization of IEEE 802.11 DCF based on Bayesian estimation of the number of competing terminals," IEEE Trans. Mobile Comput., vol. 5, no. 9, pp. 1283-1296, Sept. 2006.

[21] G. Bianchi and I. Tinnirello, "Kalman filter estimation of the number of competing terminals in an IEEE 802.11 network," in Proc. of IEEE INFOCOM, San Francisco, CA, Mar. 2003.

[22] L. Zheng and D. N. C. Tse, "Diversity and multiplexing: A fundamental tradeoff in multiple-antenna channels," IEEE Trans. Inform. Theory, vol. 49, no. 5, pp. 1073-1096, May 2003.

[23] D. Gesbert, M. Shafi, D. Shiu, P. J. Smith, and A. Naguib, "From theory to practice: an overview of MIMO space-time coded wireless systems," IEEE J. Select. Areas Commun., vol. 21, no. 3, pp. 281-302, Apr. 2003.
[24] G. Bianchi, L. Fratta, and M. Oliveri, "Performance evaluation and enhancement of the CSMA/CA MAC protocol for 802.11 wireless LANs," in Proc. of IEEE PIMRC'96, Taipei, Taiwan, Oct. 1996.

[25] Y. Lin and V. W. S. Wong, "Saturation throughput of IEEE 802.11e EDCA based on mean value analysis," in Proc. of IEEE WCNC, Las Vegas, Nevada, Apr. 2006.

[26] J. Mo and J. Walrand, "Fair end-to-end window-based congestion control," IEEE/ACM Trans. Networking, vol. 8, no. 5, Oct. 2000.

[27] A. R. Conn, N. I. M. Gould, and P. L. Toint, Trust-region Methods. Society for Industrial Mathematics, 2000.

[28] D. P. Bertsekas, Nonlinear Programming, 2nd ed. Athena Scientific, 2004.

[29] D. Bertsekas and R. Gallager, Data networks, 2nd ed. Prentice Hall, 1992.

[30] Ns-2 simulator. [Online]. Available: http://www.isi.edu/nsnam/ns/

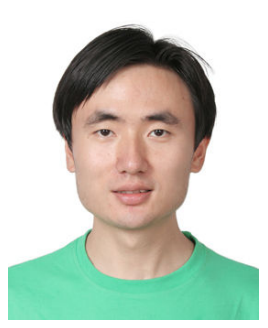

Yuxia Lin received his B.Sc. and M.Sc. degrees in applied physics and electrical engineering from Tsinghua University, Beijing, China, in 2000 and 2003, respectively. $\mathrm{He}$ is currently a Ph.D. candidate in the Department of Electrical and Computer Engineering at the University of British Columbia, Vancouver, BC. He has been granted the UBC Graduate Fellowship and the NSERC Canada Graduate Scholarship. His research interests are in the area of wireless networking with an emphasis on medium access control, cooperative communications and quality-of-

service provisioning.

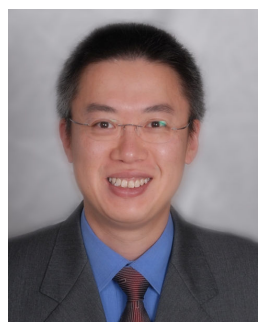

Vincent W.S. Wong (SM'07) received the B.Sc. degree from the University of Manitoba, Winnipeg, MB, Canada, in 1994, the M.A.Sc. degree from the University of Waterloo, Waterloo, ON, Canada, in 1996, and the Ph.D. degree from the University of British Columbia (UBC), Vancouver, BC, Canada, in 2000. From 2000 to 2001, he worked as a systems engineer at PMC-Sierra Inc. He joined the Department of Electrical and Computer Engineering at UBC in 2002 and is currently an Associate Professor. His research interests are in resource and mobility management for wireless mesh networks, wireless sensor networks, and heterogeneous wireless networks. Dr. Wong is an associate editor of the IEEE Transactions on Vehicular Technology and an editor of KICS/IEEE Journal of Communications and Networks. He serves as TPC member in various conferences, including IEEE Infocom'09, ICC'09, and Globecom'09. He is a senior member of the IEEE and a member of the ACM. 